\title{
Vitamin D and COVID-19: Does a Deficiency Point Towards an Unfavorable Outcome?
}

\author{
Sameer Bhimani ${ }^{1}$, Hiba Khalid ${ }^{2}$, Momina Khalid ${ }^{1}$ and Rohan Kumar Ochani ${ }^{2,{ }^{*}}$ \\ ${ }^{1}$ Jinnah Sindh Medical University, Karachi, Pakistan \\ ${ }^{2}$ Dow University of Health Sciences, Karachi, Pakistan \\ "Corresponding author: Dow University of Health Sciences, Karachi, Pakistan. Email: rohanochani@gmail.com
}

Received 2020 July 19; Revised 2020 August 10; Accepted 2020 August 16.

Keywords: Coronavirus Disease 2019, COVID-19, Biomarkers, Vitamin D, Vitamin D Deficiency

\section{Dear Editor,}

Currently, the world is grappling with the coronavirus 2019 (COVID-19) pandemic (also known as severe acute respiratory syndrome coronavirus 2), and various trials are performing to find a vaccine to combat and/or prevent its further spread. However, there is still a great deal of ground that needs to be covered, and for that reason, any promising advancement in the management of COVID-19 should be further investigated.

Known to be involved in calcium homeostasis and bone growth, vitamin D is also responsible for regulating the innate and adaptive immune system $(1,2)$. The active form of Vitamin D (i.e., 1,25-dihyroxy vitamin D) stimulates the activity of cathelicidin and the toll-like receptor (TLR), CD14, both of which are essential for the innate immune response in the lungs, thus, making active Vitamin D an important constituent of host defense (1). Additionally, regarding its ability to inhibit the expression of various inflammatory cytokines, such as tumor necrosis factor (TNF)- $\alpha$ and interferon (IFN)- $\gamma$, recent data have postulated that vitamin D can curb the "cytokine storm" speculated in COVID-19 patients (2). SARS-CoV-2 utilizes angiotensin-converting enzyme 2 (ACE2) receptors to enter ACE2-expressing cells that are mainly expressed in the lungs but are also present in other organs, which explains the wide range of complications associated with this disease (3). Uncovering a strong association between the active form of vitamin D and the renin-angiotensin system (RAS), a study conducted on rats observed that active vitamin D negatively regulates the RAS by reducing renin, ACE, and angiontensin II synthesis and induces expression of ACE23. Moreover, blocking (pro)renin receptor by active vitamin $\mathrm{D}$ helps mitigate the alveolar damage caused by lipopolysaccharide-induced acute lung injury (ALI) (3).

According to established data, there is an increased risk of severe COVID-19 likelihood and mortality in patients older than 60 years, with a history of smoking and comorbidities such as hypertension, diabetes, and cardiovascular disease $(4,5)$, all of which are associated with reduced levels of 25-hydroxyvitamin D3. Besides, a study conducted by D'Avolio et al. (6) reported remarkably lower levels of 25-hydroxyvitamin D3 in PCR-positive COVID-19 patients as compared to PCR-negative patients, suggesting the presence of a strong association between COVID-19 infection and 25-hydroxyvitamin D3 levels. Nearly 1 billion people suffer from vitamin D deficiency worldwide. Hence, it's a global public health concern across all ages and is primarily sourced through synthesis by the skin upon exposure to sunlight while the remaining is supplemented from diet (7). The occurrence of the outbreak in the fall, when 25-dihydroxyvitamin D3 levels are low due to the little or no UVB radiation, emphasizes its possible contribution to increased coronavirus infection (2). With a few exceptions, regions in the northern hemisphere have reported both higher incidence and prevalence of COVID19. A cross-sectional study conducted across Europe revealed that populations in Italy and Spain have lower mean concentrations of 25-hydroxyvitamin-D, and concurrently these countries are reported to have the highest death rates in Europe (8). Moreover, cutaneous vitamin D synthesis is reduced in dark-skinned individuals, and the elderly (7) and, interestingly, worse infection outcomes were observed in both of these demographics (8). Lastly, the implementation of stay-at-home orders and worldwide lock- 
down may have further reduced the sun exposure and may have caused intensification of vitamin D deficiency.

A systematic review of 25 randomized control trials (RCTs) revealed the overall beneficial effects of vitamin D supplementation for acute respiratory tract infections (ARTI), with the effects being stronger in daily or weekly doses with baseline 25-hydroxyvitamin D3 levels < 25 $\mathrm{nmol} / \mathrm{L}$ (9). The world is still in the preliminary stages of finding a definitive cure for COVID-19, and the importance of vitamin D in maintaining a well-developed immune system should be considered. The majority of the evidence linking vitamin D deficiency to COVID-19 comes from observational studies with mixed results. Thus, RCTs are needed to corroborate this association. If proven beneficial, supplementing vitamin $\mathrm{D}$ is straightforward due to its affordability and ease of administration.

\section{Footnotes}

Authors' Contribution: Study concept and design: SB, HK, MK, and RO. Drafting of the manuscript: SB, HK, MK, and RO. Critical revision of the manuscript for important intellectual content: SB, HK, MK, and RO. Approval of the final version of the manuscript: SB, HK, MK, and RO.

Conflict of Interests: The authors declare no conflict of interest to declare.

Funding/Support: The authors received no funding or support to perform the current study.

\section{References}

1. Hansdottir S, Monick MM, Hinde SL, Lovan N, Look DC, Hunninghake GW. Respiratory epithelial cells convert inactive vitamin D to its active form: Potential effects on host defense. J Immunol. 2008;181(10):7090-9. doi: 10.4049/jimmunol.181.10.7090. [PubMed: 18981129]. [PubMed Central: PMC2596683].

2. Grant WB, Lahore H, McDonnell SL, Baggerly CA, French CB, Aliano JL, et al. Evidence that vitamin D supplementation could reduce risk of influenza and COVID-19 infections and deaths. Nutrients. 2020;12(4) doi: 10.3390/nu12040988. [PubMed: 32252338]. [PubMed Central: PMC7231123].

3. Hadizadeh F. Supplementation with vitamin D in the COVID-19 pandemic? Nutr Rev. 2020. doi: 10.1093/nutrit/nuaa081. [PubMed: 32679589].

4. Kakodkar P, Kaka N, Baig MN. A comprehensive literature review on the clinical presentation, and management of the pandemic coronavirus disease 2019 (COVID-19). Cureus. 2020;12(4). e7560. doi: 10.7759/cureus.7560. [PubMed: 32269893]. [PubMed Central: PMC7138423].

5. Wiersinga WJ, Rhodes A, Cheng AC, Peacock SJ, Prescott HC. Pathophysiology, transmission, diagnosis, and treatment of coronavirus disease 2019 (COVID-19): A review. JAMA. 2020. doi: 10.1001/jama.2020.12839. [PubMed: 32648899].

6. D'Avolio A, Avataneo V, Manca A, Cusato J, De Nicolo A, Lucchini R, et al. 25-hydroxyvitamin D concentrations are lower in patients with positive PCR for SARS-CoV-2. Nutrients. 2020;12(5). doi:10.3390/nu12051359. [PubMed: 32397511]. [PubMed Central: PMC7285131].

7. Sizar O, Khare S, Goyal A, Bansal P, Givler A. Vitamin D deficiency. StatPearls. Treasure Island (FL); 2020. eng.

8. Laird E, Rhodes J, Kenny RA. Vitamin D and inflammation: Potential implications for severity of covid-19. Ir Med J. 2020;113(5):81. [PubMed: 32603576].

9. Martineau AR, Jolliffe DA, Hooper RL, Greenberg L, Aloia JF, Bergman $P$, et al. Vitamin D supplementation to prevent acute respiratory tract infections: Systematic review and meta-analysis of individual participant data. BMJ. 2017;356. i6583. doi: 10.1136/bmj.i6583. [PubMed: 28202713]. [PubMed Central: PMC5310969]. 